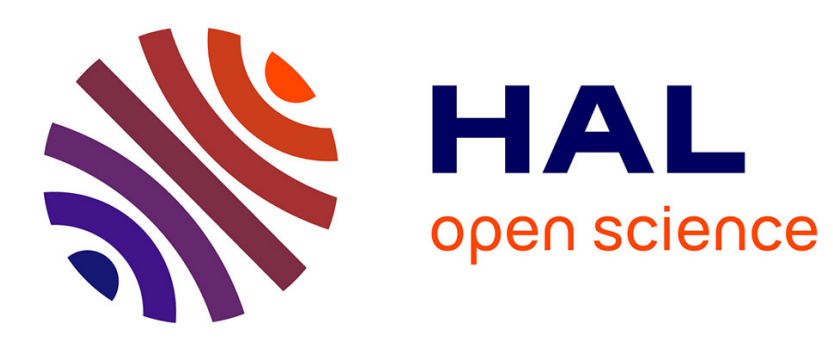

\title{
Transition laminaire-turbulent en écoulement pulsatile. Application a la circulation sanguine
}

\author{
Ph. Dantan, F. de Jouvenel, C. Oddou
}

\section{To cite this version:}

Ph. Dantan, F. de Jouvenel, C. Oddou. Transition laminaire-turbulent en écoulement pulsatile. Application a la circulation sanguine. Journal de Physique Lettres, 1976, 37 (7-8), pp.157-160. 10.1051/jphyslet:01976003707-8015700 . jpa-00231263

\section{HAL Id: jpa-00231263 https://hal.science/jpa-00231263}

Submitted on 1 Jan 1976

HAL is a multi-disciplinary open access archive for the deposit and dissemination of scientific research documents, whether they are published or not. The documents may come from teaching and research institutions in France or abroad, or from public or private research centers.
L'archive ouverte pluridisciplinaire HAL, est destinée au dépôt et à la diffusion de documents scientifiques de niveau recherche, publiés ou non, émanant des établissements d'enseignement et de recherche français ou étrangers, des laboratoires publics ou privés. 


\title{
TRANSITION LAMINAIRE-TURBULENT EN ÉCOULEMENT PULSATILE. APPLICATION A LA CIRCULATION SANGUINE
}

\author{
Ph. DANTAN, F. DE JOUVENEL et C. ODDOU \\ Université Paris VII, L.B.H.P., Tour 33/34, 4, place Jussieu, 75221 Paris Cedex 05, France
}

(Reçu le 2 mars 1976, accepté le 14 mai 1976)

\begin{abstract}
Résumé. - Les observations in vivo d'écoulements physiologiques sanguins révèlent, sous certaines conditions, la génération de bouffées de turbulence. Ce phénomène est interprété théoriquement en étudiant la stabilité des profils d'un écoulement pulsatile d'un fluide incompressible en tube circulaire rigide. On montre que le cœur non visqueux de cet écoulement instationnaire peut être instable vis-à-vis des fluctuations de vorticité.
\end{abstract}

\begin{abstract}
Studies of arterial blood flow have shown, under particular conditions, the generation of disturbed velocity waveforms having a high frequency component. A theoretical interpretation of this phenomenon is developed using the stability theory of pulsatile flow in rigid circular tube. It is shown that the non-viscous instationnary core of the flow can be unstable for the vorticity fluctuations.
\end{abstract}

La présence de bouffées de turbulence dans l'écoulement instationnaire artériel a été mise récemment en évidence, lors d'expériences in vivo [1, 2, 3], en utilisant des anémomètres à film chaud dont le temps de réponse est très court. Sous certaines conditions d'écoulement, les formes d'ondes mises en évidence dans un diagramme d'évolution temporelle de la vitesse révèlent des fluctuations à spectre relativement large (plusieurs centaines de $\mathrm{Hz}$ ) qui apparaissent dans la phase diastolique (phase de décélération ou d'écoulement retour) du cycle de la pulsation. Dans ces conditions complexes d'écoulements (non stationnaires et non harmoniques, en géométrie imparfaitement cylindrique à parois déformables), le nombre de Reynolds $\widehat{\operatorname{Re}}=2 R \hat{W} / v$ (où $\hat{W}$ est la vitesse maximum sur l'axe et $v$ la viscosité cinématique du sang) n'est pas le seul paramètre critique de la transition. Il y a lieu d'y adjoindre le paramètre de fréquence $\alpha=R \sqrt{\omega / v}$ ( $\omega$, pulsation fondamentale $\mathrm{du}$ cycle cardiaque) caractéristique du rapport des effets d'inertie (dus à la variation temporelle de la vitesse) et des effets de viscosité.

De ces expériences effectuées sur différentes espèces animales (lapin, chien, cheval) à différentes périodes du cycle cardiaque et chez l'homme dans des situations pathologiques, Nerem a déduit une relation empirique entre $\widehat{\operatorname{Re}}$ et $\alpha$, qui détermine la limite de stabilité observée : sur la figure 1 , le nombre de Reynolds critique $\widehat{R e}_{\mathrm{c}}$, déterminé par le maximum de la vitesse au centre de l'écoulement est représenté en fonction du paramètre $\alpha$ de fréquence. Pour l'écou- lement aortique chez le même chien on a pu observer un écoulement soit laminaire, soit hautement perturbé selon les valeurs de la fréquence et du volume d'éjection cardiaque. Aux grandes valeurs de $\alpha$, les observations expérimentales mettent en évidence une zone d'écoulement perturbé : la valeur minimum du nombre de Reynolds correspond à l'observation de la perturbation dans l'écoulement au pic systolique (écoulement faiblement perturbé) alors que la valeur maximum correspond à l'observation de perturbations pendant toute la phase de décélération diastolique (écoulement hautement perturbé). Bien que ce dernier nombre traduise l'apparition de la turbulence, il semble plus correct d'interpréter comme critique,

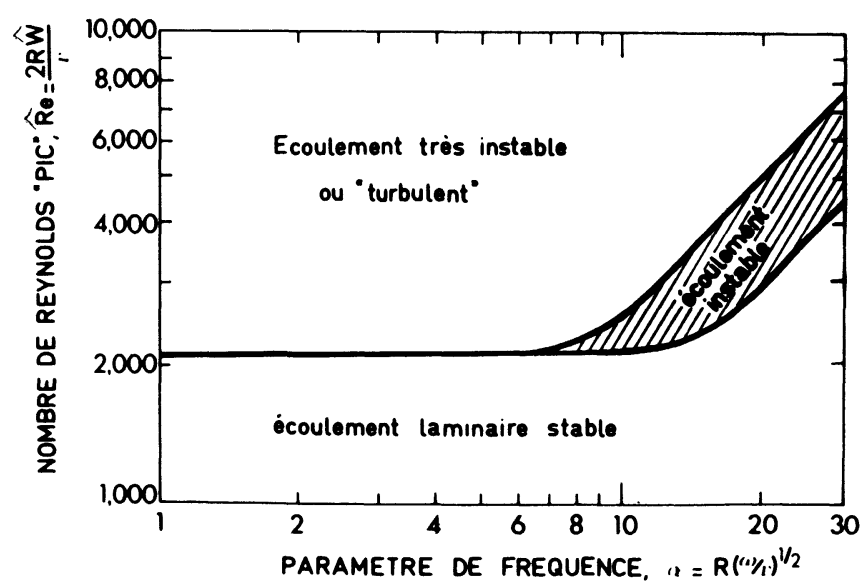

FIG. 1. - Domaines d'instabilités déterminés expérimentalement (d'après Nerem [4]) en fonction du nombre de Reynolds pic de l'écoulement sanguin artériel et du paramètre de fréquence. 
la valeur la plus faible de ces deux valeurs observées du nombre de Reynolds.

Par exemple, la limite d'instabilité dans l'aorte thoracique de chien se traduit par la relation :

$$
\operatorname{Re}_{c}=150 \alpha .
$$

Ce phénomène a été interprété en termes d'instabilité de la couche limite oscillante qui est située au voisinage de la paroi : Nerem et al. [2] ont montré que la loi empirique est en accord avec l'hypothèse de la stabilité d'une couche limite plane oscillante, d'épaisseur $\delta=(2 v / \omega)^{1 / 2}$, caractérisée par un nombre de Reynolds critique $\widehat{R e}_{\delta}=\hat{W} \delta / v=100$.

Néanmoins, les résultats de ces expériences, très délicates, restent difficiles à exploiter compte tenu des erreurs inhérentes aux expériences in vivo. En particulier, les techniques actuelles de vélocimétrie appliquées aux écoulements physiologiques ne permettent pas de déterminer avec précision le profil des vitesses de la couche limite oscillante localisée très près de la paroi. De plus, par ces techniques, on ne peut espérer accéder aux propriétés du champ des vitesses fluctuantes. C'est ainsi que les résultats expérimentaux obtenus mettent davantage en évidence un état transitoire de perturbation qu'un état de turbulence complètement développée. De nombreuses questions restent actuellement sans réponse, à la fois sur les plans théorique et expérimental : par exemple, l'influence sur la transition des différents harmoniques contenus dans l'écoulement de base. D'autre part, les écoulements physiologiques concernés présentent une composante continue (débit moyen non nul) et il y a donc lieu d'introduire outre les paramètres : nombre de Reynolds maximum $\widehat{\mathrm{Re}}$ (ou moyen $\overline{\operatorname{Re}}=2 R \bar{W} / v$, avec $\bar{W}$ vitesse débitante) et paramètre de fréquence, un troisième paramètre caractéristique $\lambda=\tilde{W} / \bar{W}$, représentant le rapport du débit instantané maximum correspondant à la composante harmonique fondamentale au débit moyen donné par la composante continue.

Dans ces écoulements physiologiques l'allure approchée du profil des vitesses peut être obtenue par la résolution des équations de Navier-Stokes en présence d'un gradient de pression pulsé (composante continue $a_{\mathrm{c}}$ superposée à une composante harmonique $\left.a_{\mathrm{h}},-|\operatorname{grad} \mathbf{p}| / \rho=a_{\mathrm{c}}+a_{\mathrm{h}} \cos \omega t\right)$. En négligeant, (i) la déformation de la paroi, (ii) les effets d'entrée et de courbure, (iii) les effets de branchement et de rétrécissement et en tenant compte des conditions de non-glissement à la paroi et d'axisymétrie du problème, l'expression du profil des vitesses (normalisées par la vitesse débitante) est la suivante [5] :

$$
\begin{aligned}
W / \bar{W}=2\left(1-x^{2}\right) & +\lambda[B(x, \alpha) \cos \omega t+ \\
& +(1-A(x, \alpha)) \sin \omega t] / C(\alpha)
\end{aligned}
$$

où

$$
\begin{gathered}
x=r / R \\
A(x, \alpha)=(\text { ber } \alpha \text { ber } \alpha x+\text { bei } \alpha \text { bei } \alpha x) /\left(\operatorname{ber}^{2} \alpha+\operatorname{bei}^{2} \alpha\right) \\
B(x, \alpha)=(\text { bei } \alpha \text { ber } \alpha x-\text { ber } \alpha \text { bei } \alpha x) /\left(\operatorname{ber}^{2} \alpha+\operatorname{bei}^{2} \alpha\right) \\
{[c(\alpha)]^{2}=[1-2 D(\alpha) / \alpha]^{2}+[2 E(\alpha) / \alpha]^{2}} \\
D(\alpha)=-\mathrm{d} B /\left.\mathrm{d}(\alpha x)\right|_{x=1} ; \quad E=\mathrm{d} A /\left.\mathrm{d}(\alpha x)\right|_{x=1}
\end{gathered}
$$

ber et bei sont les fonctions de Kelvin d'ordre 0 .

Les profils de vitesse ainsi obtenus pour des paramètres d'écoulement caractéristiques $(\alpha=15 ; \lambda=4)$ des expériences in vivo précédemment mentionnées sont donnés sur la figure 2 . Il est à noter que ces profils des vitesses adimensionnelles sont complètement déterminés par les seuls paramètres $\alpha$ et $\lambda$.

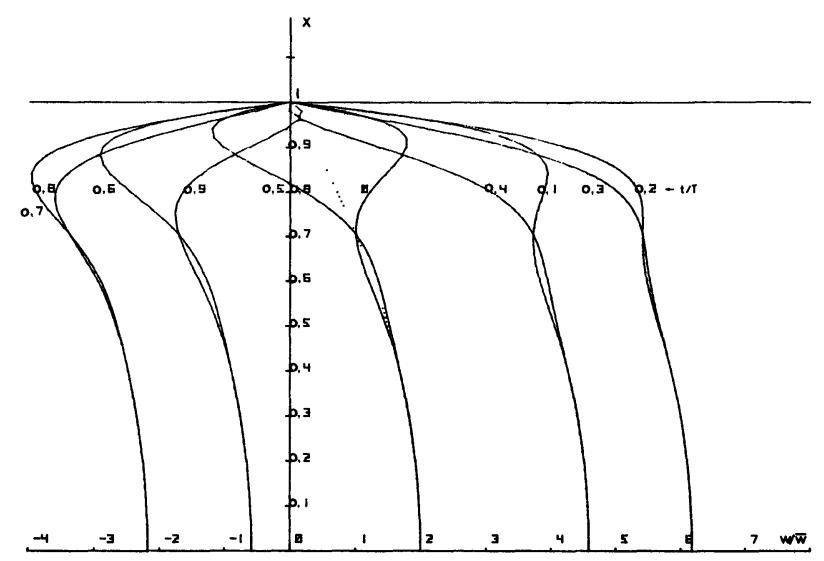

Fig. 2. - Profil des vitesses en écoulement sinusoïdal visqueux superposé à un écoulement laminaire stationnaire d'un fluide incompressible dans un tube circulairé (en pointillé, l'écoulement de Poiseuille stationnaire). $\alpha=15, \lambda=4$.

Une étude théorique de la stabilité hydrodynamique de tels profils vis-à-vis de petites fluctuations (théorie linéaire) peut être effectuée en partant du traitement linéarisé des équations de la dynamique des perturbations de vitesse $\mathbf{v}$ et pression $p$ superposées à l'écoulement de base $(V, P)$. En terme de la dynamique des perturbations de vorticité $\omega=\mathbf{r o t} \mathbf{v}$, superposées à la vorticité de l'écoulement de base $\boldsymbol{\Omega}=\operatorname{rot} \mathbf{V}\left(0, \Omega_{\theta}=-\partial_{\mathrm{r}} W, 0\right)$ les équations de Navier-Stokes linéarisées s'écrivent alors :

$$
\begin{aligned}
& D \omega / D t=\left(\partial_{\mathrm{t}}+\mathbf{V} . \mathrm{grad}\right) \omega \\
&+ \operatorname{rot}(\mathbf{v} \times \mathbf{\Omega})+ \\
&+(\boldsymbol{g} . \mathrm{grad}) \mathbf{V}+v \Delta \omega .
\end{aligned}
$$

Le débit de l'écoulement de base étant tel que la composante harmonique est grande devant la composante continue et, le nombre de Reynolds instantané maximum toujours supérieur au nombre de Reynolds critique déterminé en régime stationnaire (comme le montre la figure 1), on supposera, qu'en dehors des couches limites, la viscosité a un effet négligeable 
sur la dynamique des perturbations. Si de plus on tient compte des observations expérimentales qui mettent en évidence le fait que, dans les écoulements instationnaires, les perturbations les plus instables sont les modes axisymétriques, il est aisé de montrer à partir de (3) le rôle fondamental joué par le terme de production des fluctuations azimutales de vorticité [6] :

$$
-u r \partial_{\mathrm{r}}\left(\Omega_{\theta} / r\right)=u\left[W^{\prime \prime}-W^{\prime} / r\right]
$$

où $u$ désigne la composante radiale des perturbations de vitesse. Il en résulte que la condition de stabilité marginale, compte non tenu des effets dissipatifs dus à la viscosité moléculaire, est obtenue lorsque ce terme est nul, soit :

$$
W^{\prime \prime}-W^{\prime} / r=0 .
$$

Cette condition est à rapprocher du critère d'instabilité concernant le point d'inflexion des profils de vitesse des écoulements plans établis par Rayleigh [7].

Un autre critère d'instabilité généralisant la condition de vorticité maximum de l'écoulement de base établie dans le cas des écoulements plans par Hoiland [8] a été récemment développé pour des écoulements axisymétriques instationnaires [9]. Il fait jouer, à la présence de points $\mathrm{P}$ dans le diagramme position radiale-temps, où la condition (5) est satisfaite et où, de plus :

$$
W=0,
$$

un rôle fondamental dans l'apparition des instabilités, comme on a pu d'ailleurs l'observer expérimentalement [10]. Cette deuxième condition est nécessaire pour qu'une perturbation de fréquence donnée puisse croître car, l'écoulement de base dépendant du temps, les termes de convection conduisent généralement à une dissipation par couplage non linéaire et changement de fréquence.

Dans ces conditions (effets de la viscosité négligeables et seules les perturbations symétriques étant considérées), la présence dans l'écoulement de points $P$

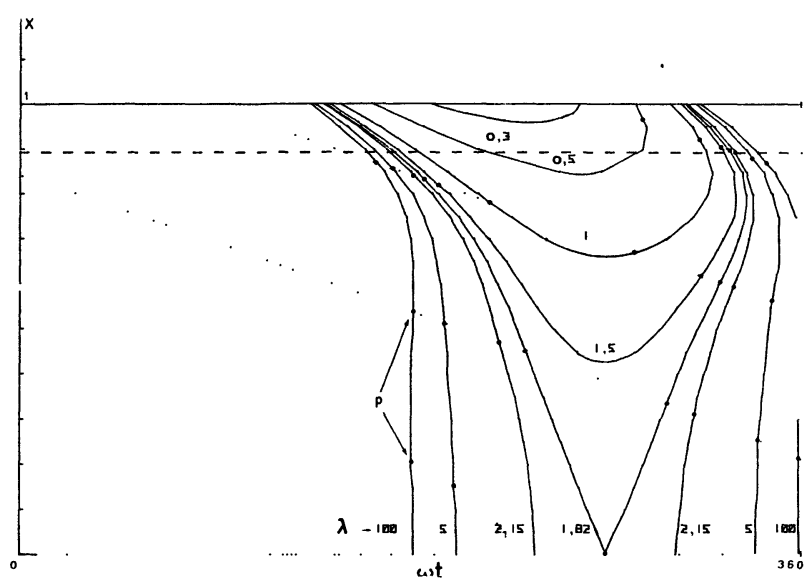

FIG. 3. - Lieu des points de vitesse nulle (— relation (6)) et des points de production nulle des fluctuations de vorticité $(. .$. relation (5)). Points P, ----- couche limite. $\alpha=15$. où (5) et (6) sont simultanément satisfaites est déterminante pour l'apparition d'instabilité et le passage d'un état laminaire à un état instable. Ainsi, sur la figure 3, pour $\alpha=15$, les équations (5) et (6) appliquées à l'écoulement pulsatile donné par (1) ont été tracées dans le diagramme $(r, t)$ ainsi que les points $P$. On peut noter que sur l'axe du tube la relation (5) est constamment vérifiée alors qu'à la paroi, d'après l'hypothèse de non-glissement, (6) est toujours satisfaite. D'autre part, on doit remarquer aussi que seule la partie pulsatile de l'écoulement intervient dans la relation (5), car l'écoulement de Poiseuille stationnaire satisfait partout cette relation. L'étude systématique de tels diagrammes, lorsque les paramètres $\alpha$ et $\lambda$ varient, met en évidence les caractéristiques suivantes :

(i) Aux faibles valeurs du paramètre de fréquence $(\alpha<10)$ l'allure des profils est telle qu'un nombre restreint de points $P$ apparaissent dans l'écoulement. La couche limite oscillante (où les effets de la viscosité sont prépondérants) étant de l'ordre de grandeur des dimensions diamétrales du tube, le seul point satisfaisant les conditions imposées théoriquement est donc situé sur l'axe du tube et l'instabilité est alors déclenchée par sa présence. Ceci implique, dans ces conditions, que l'écoulement devienne instable dès qu'apparaît un écoulement retour sur l'axe. La vitesse en ce point a pour expression :

$$
W / \bar{W}=2+\lambda \rho(\alpha, 0) \cos (\dot{\omega} t-\delta(\alpha, 0)) / C(\alpha)
$$

où

$$
\begin{aligned}
& \rho(\alpha, 0)=\left[(1-A(\alpha, 0))^{2}+(B(\alpha, 0))^{2}\right]^{1 / 2} \\
& \delta(\alpha, 0)=\operatorname{Arctg}[(1-A(\alpha, 0)) / B(\alpha, 0)]
\end{aligned}
$$

Les valeurs des paramètres $\alpha, \lambda$ pour lesquelles $W=0$ sur l'axe du tube satisfont donc la relation :

$$
\cos (\omega t-\delta(\alpha, 0))=-2 C(\alpha) / \lambda \rho(\alpha, 0) .
$$

Cette relation admet une solution si :

$$
2 C(\alpha) / \lambda \rho(\alpha, 0) \leqslant 1 .
$$

L'inégalité stricte donne 2 racines correspondant aux intersections des courbes ouvertes, $W=0$, avec l'axe (cf. Fig. 4), courbes d'autant plus séparées que $\lambda$ est grand (comme le montre la relation (10)).

L'égalité correspond à la situation où la courbe $W=0$ est tangente à l'axe (racine double en $\omega t$. de la relation (9)). Elle correspond donc à la valeur critique $\lambda_{\mathrm{c} 1}$ telle que :

$$
\lambda_{\mathrm{c} 1}=2 C(\alpha) / \rho(\alpha, 0)
$$

dont la variation en fonction de $\alpha$ est donnée sur la figure 4 (Courbe A).

(ii) Aux grandes valeurs du paramètre de fréquence $(\alpha>10)$, les courbes $W^{\prime \prime}-W^{\prime} / r=0$ sont des droites d'autant moins écartées et inclinées que $\alpha$ est grand. 


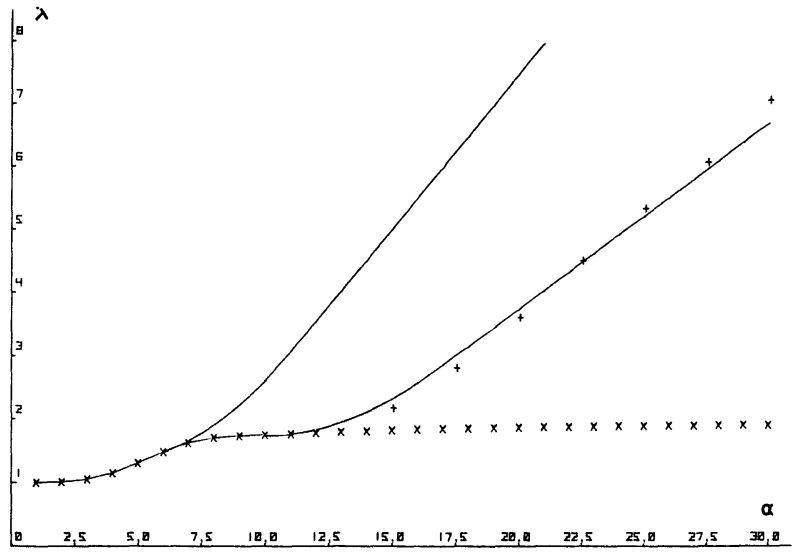

Fig. 4. - Comparaison des résultats expérimentaux [4] et théoriques présentés ici.

$\times \times \times \times A$, points $P$ sur l'axe.

$++++B$, points $P$ à la frontière de la couche limite. $C$, résultats expérimentaux.

Il en résulte un grand nombre de points $P$ dont certains, suivant les valeurs de $\lambda$, sont situés dans la couche limite. Il existe alors une valeur critique $\lambda_{c 2}$ au-delà de laquelle tous les points $P$ sont en dehors de cette couche. Les critères d'instabilités concernant la présence de ces points ayant été établis dans le cadre d'une théorie de fluide non visqueux, on est amené à déterminer, pour chaque valeur de $\alpha$, l'épaisseur $\Delta$ de cette couche et à chercher la valeur critique de $\lambda$ pour laquelle aucun point $\mathbf{P}$ n'apparait pour $x>1-\Delta$

La détermination de l'épaisseur moyenne $\Delta$ de la couche limite oscillante a été effectuée, pour différentes valeurs de $\alpha>15$, en relevant les profils d'écoulement harmonique à différents temps dans la période (18 dates également espacées entre 0 et $\pi$ ). Il a été ensuite fait une moyenne statistique des ordonnées des maxima de vitesse compris entre la paroi et l'ordonnée correspondant à la vitesse maximum dans le temps. Cette détermination est d'autant plus précise que $\alpha$ est grand mais pratiquement impossible pour $\alpha<15$. Les valeurs ainsi obtenues sont comprises entre les courbes $\sqrt{2} / \alpha$ et $2 / \alpha$, généralement admises comme épaisseur approchée de la couche limite oscillante [11]. La valeur de $\lambda_{\mathrm{c} 2}$ est déterminée expli- citement à partir de (1), (5) et (6) et $x=1-\Delta$ (courbe B de la Fig. 4).

La confrontation de cette théorie avec les résultats des, expériences précédemment citées a été faite en supposant que les fluctuations de l'écoulement moyen stationnaire ne sont amplifiées que si les conditions (5) et (6) sont satisfaites. Les résultats expérimentaux sont interprétés en faisant l'hypothèse que le débit moyen reste constant lorsque les conditions d'écoulement, contrôlées par la fréquence et le volume d'éjection cardiaque (fixant $\alpha$ et $\widehat{\mathrm{Re}}$ ), varient. Compte tenu des manipulations effectuées [12] pour faire varier la fréquence cardiaque et la vitesse maximum (injections intraveineuses d'isoprénaline, de propanolol, de pentobarbitone ou stimulation nerveuse), l'hypothèse d'invariance de la résistance périphérique, et donc du débit moyen, n'est qu'approximativement vérifiée. Elle conduit à un nombre de Reynolds moyen $\overline{\mathrm{Re}}$ voisin de 500 (vitesse débitante de l'ordre de quelques dizaines de $\mathrm{cm} / \mathrm{s}$ ) et, compte tenu des définitions précédentes des nombres de Reynolds maximum $\widehat{\mathrm{Re}}$ et moyen $\overline{\mathrm{Re}}$, il en résulte la relation :

$$
\widehat{\operatorname{Re}}=\overline{\operatorname{Re}}[2+\lambda \rho(\alpha, 0) / C(\alpha)] .
$$

Etant donné $\alpha$, cette relation permet de traduire les résultats expérimentaux (Fig. 1) donnés par les courbes de stabilité marginale $\operatorname{Re}(\alpha)$ en courbes $\lambda(\alpha)$ (Courbe C, Fig. 4). Dans la limite de variation des paramètres des écoulements physiologiques expérimentalement étudiés, et compte tenu du modèle simplifié adopté ici, on note une très bonne concordance entre résultats expérimentaux et théorie.

L'explication théorique donnée ici diffère sensiblement des interprétations précédentes [12]. En effet, celles-ci n'avaient pas considéré l'influence du paramètre $\lambda$ et avaient porté l'accent sur la stabilité de la couche visqueuse instationnaire. Contrairement à cette thèse nous interprètons le phénomène d'instabilité en écoulement pulsatile comme lié au comportement du cour non visqueux de l'écoulement. Une étude expérimentale détaillée (actuellement en cours) du champ d'écoulement pulsatile sur modèle hydrodynamique devrait permettre d'étayer cette thèse.

\section{Bibliographie}

[1] Schultz, D. L. et al., In Ciba Foundation Symposium on Circulatory and Respiratory Mass Transport (London Churchill) 1969 , pp. 172-99.

[2] Nerem, R. M., Seed, U. A. and Wood, W. B., J. Fluid. Mech. 52 (1972) 137

[3] Seed, W. A. and Thomas, J. R., In Fluid Dynamic Measurements in the Industrial and Medical Environments (Leicester University Press) 1972, p. 298.

[4] Nerem, R. M., Paper presented as an invited Lecture to the Laennec Society of the American Heart Association 47th. Scientific Sessions Meeting, Dallas, Texas November 18-21 (1974).
[5] UCHILDA, S., Z.A.M.P. 7 (1956) 403

[6] Betchov, R. and Criminale, W. O. Jr., In Stability of Parallel Flows (Academic Press) 1967, p. 228.

[7] Rayleigh, In Scientific Papers I (1880) 474.

[8] Hoiland, E., Geophys. Publ. Norske Vindenskaps. Akad. Oslo 18 (1953) 1.

[9] Pelissier, R., Clarion, C., J. Fluid. Mech. 70 (1975) 59.

[10] Sergeev, S. I., Fluid Dyn. 1 (1966) 121.

[11] SCHLICHTING, Boundary Layers theory (Mc Graw Hill Book Company NY) 1968.

[12] Nerem, R. M. and SeED, W. A., Cardiovasc. Res. 6 (1972) 1-14. 